\title{
IDENTIFICAR LOS TONEMAS DEL ESPAÑOL CON Y SIN DISTORSIÓN DE LA INFORMACIÓN SEGMENTAL
}

ALBERTO CARCEDO GONZÁlEZ Universidad de Turku (Finlandia)

\section{INTRODUCCIÓN}

Aunque en principio y en igualdad de condiciones cualquier ser humano tiene las mismas posibilidades fónicas, el aprovechamiento que de ellas se hace en las distintas lenguas es diferente. De ello se deriva que los hablantes de una determinada lengua presenten en su proceso de aprendizaje de una nueva - si prescindimos de las variantes causadas por el sexo, la edad, la procedencia geográfica (dialectal), social, cultural, etc.- unos rasgos comunes que los diferencian de quienes, aprendiendo la misma lengua, tienen como materna otra diferente. En líneas generales, a la hora de analizar las divergencias que la lengua empleada por un extranjero presenta respecto a la del nativo podemos establecer dos planos perfectamente diferenciados: el fonológico y el fonético. Las desviaciones que se produzcan en el primero no afectan al mensaje (aunque no por ello deberían ser olvidadas por el profesor); las que se produzcan, sin embargo, en el segundo —el fonologico- revestirán obviamente mayor gravedad porque pueden afectar a la comunicación: la sustitución de un fonema por otro puede provocar la confusión de dos palabras entre sí, y dar lugar con ello a una interpretación errónea del mensaje. En la entonación, al igual que en los segmentos, junto a aquellas variaciones que carecen de relevancia 
desde el punto de vista semántico, como son las diferencias tonales que aparecen en el interior de la frase, otras - las que se registran en el tramo final del grupo fónico- tienen verdadera importancia, pues el valor que cobre el enunciado según aparezcan unas u otras será afirmativo, interrogativo, etc. '. Estas consideraciones que son válidas para el español no lo son necesariamente para todas las lenguas. En finés, por ejemplo, la entonación parece cumplir un papel mucho menos relevante que en español. Tal vez ello explique las dificultades que los hablantes de aquella lengua manifiestan a la hora de reproducir los esquemas entonativos característicos de la nuestra. $Y$ producir está, sin duda, íntimamente ligado a discriminar. ¿Son capaces nuestros alumnos de percibir las diferencias existentes entre los diferentes patrones melodicos del español? En este trabajo, junto a unas consideraciones iniciales sobre la entonación del finés y el español, así como las diferencias teóricas que separan a ambos sistemas, damos cuenta de los resultados obtenidos mediante un test de discriminación con dos diferentes pruebas. Ello nos ha permitido saber cuáles son las inflexiones melodicas del español que causan las mayores dificultades a aquellos hablantes, los tonemas del español hacia los que se producen habitualmente las desviaciones, el tipo de identificación que de los tonemas menores se hace y, por último, el grado de incidencia que la comprensión de la parte segmental desempeña en una correcta identificación del elemento suprasegmental.

\section{Sobre los tonEMAS DEL ESPAÑol Y EL FINÉs}

Siendo la entonación una consecuencia de la tensión de los músculos de la laringe, está presente en toda manifestación hablada. Esta tensión se aprende a controlar y presenta diferencias entre las diversas lenguas, lo que motiva que, de la misma manera que en el aprendizaje de una lengua extranjera se co-

' Para la mayoría de los estudiosos del español la parte realmente importante de la entonación está localizada en el tramo final del enunciado. La melodía del resto de la oración sería fonológicamente irrelevante, sirviendo para expresar variables dialectales, sociales y expresivas. Así, para AlarCos LloRACH (1994: 51), «Lo verdaderamente pertinente en la entonación es esa inflexión final; mientras que los tonos anteriores pueden fluctuar sin sujeción a un esquema rígido. Así quedan libres para sugerir los estados de ánimo o las intenciones del hablante, como recurso expresivo de la comunicación o como señal de su pertenencia a un grupo social o regional determinado». QUILIS y FERNANDEZ (1979: 164-165) son de la misma opinión: «En el comportamiento melódico de la frase [...] hay que distinguir cuidadosamente entre: $1 .^{\circ}$, las variaciones tonales que existen en el interior de la frase y que pueden atribuirse a peculiaridades regionales, individuales $u$ ocasionales, y $2 .^{\circ}$, las variaciones tonales que aparecen al final del grupo fónico, que son significativas». 
meten errores en la articulación de los sonidos, se produzcan también en la entonación.

Y es que, como nos dice Lieberman (1967: 44), la entonación se aprende antes que los otros rasgos lingüísticos, pertenece a un nivel de conocimientos anterior al de los rasgos fonéticos - que se incorporan más tarde- y es, por tanto, más difícil de desterrar después de que el aprendiz de una lengua extranjera ya posee un buen nivel en la pronunciación de los segmentos. Así, Lorenzo (1994: 18) considera la entonación «el elemento lingüístico más arraigado e inextirpable».

Del importante papel que la entonación desempeña son ilustrativas estas palabras de Navarro Tomás:

Influyen más en la fisonomía fonética de un idioma las cadencias peculiares que las circunstancias relacionadas más concretamente con la articulación y timbre de sus sonidos.[...] el conocimiento de la entonación representa un valioso y decisivo progreso en la difícil conquista e interpretación del modo de ser que el acento de ese idioma representa. No se penetra enteramente en el dominio de una lengua mientras no se conoce la intimidad de su entonación (1974: 8).

Aunque en su lengua parece ser menor, también los estudiosos finlandeses coinciden con los españoles en acentuar, frente a aquéllos que se la restan en favor de los segmentos, la importancia de la entonación, bien por considerarla un requisito indispensable para la buena consecución de la comunicación (Lehtovaara, 1978: 74), bien para conseguir una buena pronunciación. Hirvonen, por ejemplo, dice:

Correct stress and intonation [...] which are very closely connected both physiologically and physically- probably contribute more to a native-sounding pronounciation than any other phonetic features: $A$ wrong intonation contour immediately betrays a foreigner, no matter how good his segmental sounds are (1970: 85-86).

La propia naturaleza de ese rasgo suprasegmental y la gran vaguedad de conceptos que sobre él imperan hacen de su estudio una tarea ardua y complicada. Como dice Quilis (1981: 340), «es imposible poder captar en una definición toda la complejidad sustancial, formal y funcional de la entonación». En la misma dirección se manifiesta para el finés Karlsson cuando afirma:

Parece ser caracteristica de los rasgos prosódicos una cierta ambigliedad [...] por medio de los rasgos prosódicos es posible expresar 
valores y estados de ánimo, pero es difícil establecer para ellos una clasificación categórica, y por ello no resulta fácil dar cuenta de ellos $[\ldots]^{2}$ (1983: 165).

La mayoría de las lenguas distingue tres partes en el grupo fónico o unidad melódica: inicia, medial y final. Parece ser general, además, el ascenso de la parte inicial de la curva de entonación, variando de una lengua a otra en el grado y la duración de la subida. El cuerpo central de la entonación presenta diferencias mucho más acusadas entre las distintas lenguas, caracterizándose la del español —especialmente el de Castilla—, según Gili Gaya (1975: 60), por una tendencia a la uniformidad: «las ondulaciones de voz se producen en esta parte del grupo fónico con pequeños intervalos», contrastando en este sentido con la amplitud que presentan las inflexiones del italiano y el portugués, o la mayor movilidad del francés, el inglés y el alemán. Las subidas y descensos de la parte final de la curva melódica -y los valores que conllevan-, si bien coinciden en muchas lenguas, no podemos decir que tengan validez universal. Las similitudes que podemos observar entre diferentes lenguas - los tonos agudos suelen asociarse con estados de ánimo de mayor emoción, con enunciados incompletos que reclaman la atención del interlocutor, preguntas, etc., mientras que los enunciados completos, el final del mensaje, el desinterés, el desánimo, etc. van acompañados, por el contrario, de tonos más bajos- no bastan para generalizar. Y es que todos nos damos cuenta de la peculiar forma en que «cantan» al hablar los naturales de otros países o incluso del nuestro propio cuando proceden de otra región. No percibimos, sin embargo, en nuestra forma de hablar que «cantemos». Los finlandeses de lengua sueca consideran que los suecos «cantan» cuando hablan. Esa misma opinión tienen los suecos de sus vecinos finlandeses suecohablantes. La diferencia entre los patrones entonativos de esas dos variantes del sueco es notoria. Además, parece claro que las lenguas suelen tener, independientemente de los ascensos y descensos a los que ya hemos hecho alusion, una entonación media característica: el francés, por ejemplo, tiene una entonación aguda, mientras que en alemán predomina la grave; los italianos presentan un registro más variado en comparación con los franceses, etc. La entonación está sometida, además, a otro tipo de factores: la cultura, el carácter, etc. La entonación de estos pueblos del sur de Europa parece tener unas variaciones de la línea melodica más acusadas que las lenguas de los pueblos del norte. Del español se opina, por ejemplo, que las inflexiones tonales «son más profundas, más dignas, más varoniles y más segu-

${ }^{2}$ Es nuestra la traducción al espafiol de ésta y las otras citas que a lo largo del trabajo han sido recogidas del finés. 
ras que el resto de las lenguas románicas» (livonen 1987: 34). El uso que de las posibilidades entonativas hacen los distintos pueblos varía notablemente.

Variantes individuales - por razones fisiologicas, psicologicas, sexo, etc. - o geográficas y dialectales —en virtud de los hábitos peculiares de cada lugar, comarca, país - aparte, el campo de entonación - amplitud tonal del intervalo en que se desarrollan los movimientos de la voz- en el español estándar suele comprender un promedio, según Navarro Tomás (1974: 26-28), de dieciséis semitonos, estando el tono normal en la voz masculina entre E:mi ${ }_{2}$ y F.fa ${ }_{2}$. El mismo estudioso considera esenciales para la caracterización de un idioma el tono y la amplitud del campo de entonación:

No se acierta a dar a la pronunciación de una lengua su propio carácter mientras no se ajusta al campo de entonación y al tono normal que le corresponde (Navarro Tomás, 1974: 29).

La entonación del finés en general es considerada monótona. La curva melódica no registra en la lengua corriente grandes alteraciones. Monola (1976: 38), por ejemplo, constata que en contadísimos casos las variaciones superan la octava. Lieko (1992: 109), por su parte, afirma: «en finés la escala entonativa es mucho más reducida que en muchas otras lenguas». Hakulinen (1979: 33) menciona entre las características típicas del finés: hablar bajo, empleo de registros graves y de pequeños intervalos, así como falta de énfasis de tipo emocional. El importante papel de la entonación en las lenguas indoeuropeas es, como afirma Karlsson (1981: 96), el que cumple en finés todo un sistema de partículas —《ko», «pa», «kin», «hän»— que pueden desempeñar las funciones que en otras lenguas realiza la entonación, y con valores tan diversos como interrogación, insistencia, confirmación, etc.

Aunque de los índice físicos del acento - tono, intensidad y cantidad-, el tono parece ser el componente fundamental, la melodía también se ve afectada por las variaciones de intensidad y la duración de los distintos sonidos. Alarcos Llorach (1994: 50) aduce como ejemplo de la importancia de éstos el habla cuchicheada, donde la ausencia de vibraciones glotales impide que aparezca el tono, y, sin embargo, los rasgos diferenciales de cada contorno siguen siendo reconocibles gracias a la intensidad y la duración, que reciben en compensación un refuerzo.

Han de tenerse en cuenta, además, una serie de factores, por ejemplo, la relación que frecuencia del fundamental y calidad de la vocal mantienen, ya que las vocales más altas tienen un fundamental más alto (Quilis, 1981: 356459), y el tipo de consonante que precede o sigue: las frecuencias más altas aparecen después de las consonantes sordas; las más bajas, después de las so- 
noras, etc. La frecuencia del fundamental presenta unas diferencias notables entre los hombres, las mujeres y los niños. Por último, es importante la magnitud del cambio de frecuencia. Los estudiosos difieren en este punto, alternando los mínimos entre $6 \mathrm{~Hz}$ y $40 \mathrm{~Hz}$ para que la alteración en el nivel de frecuencia pueda ser percibida como un cambio en la entonación.

También entre los estudiosos finlandeses es generalmente compartida la opinión de que el índice más importante lo constituyen las alteraciones en la frecuencia del primer armonico, aunque como dice Lieko (1992: 106), «puede deberse también a otros factores, además de los cambios en la frecuencia del fundamental» ${ }^{3}$. Karlsson comenta al respecto:

Está claro que físicamente la intensidad y la altura tonal son factores diferentes. Son perceptibles además, como elementos distintos fuerza y altura. En la práctica, sin embargo, marchan paralelamente en la mayor parte de los casos (1983: 168). ${ }^{4}$

Penttilä, por su parte, afirma:

Entonación y acentuación en finés parecen marchar paralelamente, de forma que a una mayor fuerza acentual corresponde normalmente un nivel tonal más alto. Al menos está claro que la altura tonal de la sílaba portadora del acento principal es mayor que la de las silabas con acento secundario o las átonas. [...] También la intensidad de la sílaba y la entonación actúan paralelamente (1958: 2).

En cuanto a las funciones de la entonación se refiere, Quilis (1981: 376395) establece tres diferentes planos a la hora de estudiar las funciones de la entonación: linguiístico, sociolinguiústico y expresivo, y dos tipos de funciones: lingüísticas y no lingüísticas. En el primer nivel diferencia funciones exclusivamente lingüísticas: la integradora (una sucesión de unidades apelativas se convierten mediante ella en unidades comunicativas: enunciado), la distintiva (distingue un enunciado afirmativo de uno interrogativo) y la delimitadora o

${ }^{3}$ HIRVONEN (1970: 28) afirma: «In Finnish the intensity curve generally follows the melodic curve in broad out line».

4 De cara a estudiar la influencia de los diferentes componentes de la entonación en la interpretación del mensaje es interesante el trabajo de Helin, Nieminen, PETHMan, VilpPula y VäisäNEN (1985), en el que mediante un sintetizador acústico, y realizando alteraciones en el tono fundamental, la intensidad y la velocidad de elocución, intentan producir impresiones diferentes. Los resultados alcanzados ponen de manifiesto evidentes regularidades entre la forma prosódica y el nivel semántico. 
demarcativa (segmenta el discurso en un cierto número de unidades). En el segundo plano, la entonación proporciona información intrínseca sobre el individuo, además de la propiamente sociolinguística -l grupo al que pertenece, origen geográfico, etc.-, con lo que cumple también una función identificadora. En el tercer nivel, por último, distingue la función expresiva, pues la melodía informa sobre las actitudes y reacciones del locutor en el momento del habla.

La entonación en finés no cumple la función distintiva desempeñada por la entonación en español, o es sólo potencial, pues los enunciados en los que podría ser operativa - por las diferencias de tono a las que ya hemos hecho alusión- la pregunta ya viene marcada por medios gramaticales. Karlsson (1983: 173) habla de la «importante función delimitadora», pues «en general una entonación descendente [...] es señal para el oyente de cambio en el turno de intervención: el hablante está concluyendo la suya. Por ello, un tonema de suspensión constituye señal de que la intervención ha quedado inconclusa o de que el hablante tiene intención de continuar hablando». La entonación, pues, segmenta el discurso en frases. Además, la entonación puede ser usada para dividir las frases en unidades más pequeñas, manteniendo el tono en un nivel relativamente alto. Hirvonen, por su parte, lo ve así:

When the speaker wants to indicate to his listener that the end of the clause is not the end of the sentence, he uses a breath-group in which the falling terminal contour of the normal breath-group is replaced by a level final contour (Hirvonen, 1970: 39).

Si la frase comienza con una partícula subordinante, sin embargo, la entonación pierde importancia e incluso no se utiliza en algunos casos:

A case in point is an initial clause beginning with a subordinate conjunction. In such cases the special intonation contour is not so important and is not always used.

La función de la entonación, por otra parte, para Hirvonen (1970: 8), es tanto comunicativa —es portadora del significado de una unidad rítmica determinada, al tiempo que señala su final-como expresiva ${ }^{5}$, en cuanto es expo-

5 En relación con la función expresiva de la entonación en finés, LEHTONEN (1971) analiza el tipo de información proporcionada por la línea melódica sobre la actitud del hablante, así como la forma de medirla, definirla y describirla acústicamente. También debe destacarse en relación con la expresividad el fenómeno de enfatización señalado por PENTTILÄ (1958), que consiste en dotar a ca- 
nente de sentimientos y actitudes. Para poder interpretar correctamente el aspecto afectivo del enunciado es necesario, por tanto, conocer, al menos en alguna medida, los rasgos que caracterizan la forma de hablar del sujeto en cuestión, su idiosincrasia.

En cuanto a las inflexiones tonales del enunciado se refiere, Navarro Tomás distingue cinco para el español: «La entonación española en el modo de enunciación corriente utiliza con valores definidos cinco tonemas distintos: cadencia, semicadencia, anticadencia, semianticadencia y suspensión» (1971: 6-7). Quilis y Fernández (1979: 165) los reducen a tres: descendente (incluye cadencia y semicadencia), horizontal y ascendente (con anticadencia y semianticadencia), desde el punto de vista fonologico. Alarcos Llorach (1994: 52) también prefiere ver semicadencia y semianticadencia como variantes de la cadencia y de la anticadencia, respectivamente, «condicionadas por el contraste alternativo de los tonos dentro de la secuencia», que «sólo aparecen en interior de enunciados, pues ante pausa terminal se identifican con cada uno de los otros dos tonemas de cadencia y anticadencia”. Alcina y Blecua (1975: 471), por su parte, prefieren ver los contrastes menores como variantes de la suspensión. Existen, pues, diferencias de opinión en cuanto a la distribución de esos dos últimos tonemas.

Por lo demás, aunque las diferencias entre los esquemas entonativos son muy amplias según los diferentes niveles lingüísticos y las peculiaridades dialectales dentro del dominio hispanohablante, si nos atenemos al español estándar —en este caso el español peninsular - y seguimos la clasificación realizada por Navarro Tomás (1974), nos encontramos con cuatro grandes grupos: enunciativo (afirmativo, enumerativo, complemento circunstancial, aposición predicativa, vocativo, paréntesis, coordinación y subordinación), interrogativo (pregunta absoluta, relativa, restrictiva, aseverativa, pronominal, reiterativa, exclamativa, hipotética, alternativa y de varios grupos), volitivo y emocional.

En cuanto al finés se refiere, Ivonen (1987: 238) opina que la característica prosódica más conocida es la entonación de las frases enunciativas o de constatación, con una línea melódica en lenta inflexión descendente hasta la última sílaba acentuada, a partir de la cual el descenso es mucho más rápido, siendo la última sílaba muchas veces cuchicheada. En palabras de Hirvonen,

Complete statements in Finnish are characterized by a slowly descending scale of stressed syllables that begins at or bellow the

da sílaba de la palabra -especialmente la segunda- de un tono superior al normal. El resultado es que ésta tendría un tono igual o similar al de la primera sílaba. Cuando ésta se percibe como si fuese portadora del acento principal se debe, según PENTTILA (1958: 19) a que su entonación es ascendente-descendente. 
middle of the speaker's voice range. The unstressed syllables inbetween are spoken on a pitch somewhat lower than those of the adjacent stressed syllables. After the last stressed syllable the pitch falls sharply, resulting in a creaky voice at the very end. Sometimes the last syllable is whispered. The low, usually aperiodic fundamental frequency of the last syllable cannot be accurately measured in mingograms (1970: 26).

Karlsson (1983: 170) constata el ascenso que se produce en esa línea descendente en la palabra clave —que él llama «informatiofokus»—, portadora de la información más importante de la frase, y que está caracterizada no sólo por una mayor altura tonal sino también por ser portadora del principal acento de la frase.

El lento descenso del enunciado, como Suomi (1975) ha demostrado, aparece tanto en las frases completas como en las incompletas. La única particularidad que diferencia a las últimas es la ausencia de ese pronunciado declive final. Y es que, según Hirvonen (1970: 29), la última sílaba tónica es tan alta o más que la primera. Después del núcleo desciende, pero sólo ligeramente; a partir de aquél el nivel tonal se mantiene hasta el final. Este tipo de enunciados cuyo sentido está incompleto representan el otro modelo de entonación frecuente en el finés y expresa continuación o interrupción. Señal de ello, para Wiik (1981: 118), lo constituye el hecho de que «en finés, como en muchas otras lenguas, la línea melódica no registra un descenso tan acusado como el que aparece ante el final del enunciado». Normalmente aparece transcrita con coma, y significa pausas, en las que la entonación se mantiene relativamente estable y alta. Este tipo de pausas no son necesariamente dependientes de la sintaxis. Desde el punto de vista delimitativo -aspecto que destaca Karlsson (1983: 173) — una inflexión descendente de la curva melodica significa final de la intervención, mientras que si el tonema final se mantiene suspendido ${ }^{6}$ significa que la frase está incompleta, lo que es válido también para el español.

La pregunta en finés está caracterizada por la presencia de los sufijos ఒko», - kö», las partículas interrogativas, algunos lexemas del tipo «vai» (conjunción disyuntiva en oraciones interrogativas) y algunas partículas con valor de pregunta, como «entä» ( ¿y ... ?). Sin embargo, aunque no es frecuente, la pregunta puede formularse, como nos dice livonen (1987: 240), sin necesidad de elemento

6 En este sentido, según AALTONEN y WiK (1979), el carácter inconcluso del mensaje no s6lo se manifiesta con un tonema de suspensión sino también con una mayor altura tonal al comienzo de la frase. 
gramatical alguno. ¿Es posible en finés, entonces, servirse de medios pros6dicos para expresar la pregunta? La respuesta parece ser negativa, pues, como apunta Kallioinen (1968), las entonaciones ascendentes presentes en algunas frases interrogativas encierran otro tipo de valores, principalmente de tipo expresivo: sorpresa, apelación cortés al interlocutor, etc., o se deben a la influencia de lenguas extranjeras -es el caso de las inflexiones que pueden oírse principalmente en Helsinki y alrededores-, pero no debe concedérsele un valor distintivo a esa entonación ascendente en las frases interrogativas, puesto que su aparición se limita a esos casos aislados. Es, por tanto, considerada extraña al finés, e incluso duramente criticada por algunos (Ahonen-Mäkelä 1975: 55). Es innegable, sin embargo, la aparición de una curva tonal ascendente en algunos casos como los monosílabos «niin» (sí/ya), «vai» (o) y «no» (pues...) (livonen 1980), así como en locuciones del tipo «minä vai?» (¿yo?), «eikö niin?» (¿no es cierto?), «eikö totta?» (¿verdad?), «eikö vaan?» (¿verdad que sí?), etc., además de las formas habituales de lengua hablada con «eiks...», forma abreviada de «eikö» (¿no...?). También se han observado entonaciones ascendentes en los dialectos de algunas regiones del norte y del este: Rovaniemi, Torniolaakso, Jällivaara, etc. En estos casos se ha creído ver como causa la influencia de lenguas laponas o del sueco. Además, aunque no es siempre, también puede aparecer entonación ascendente en finés en lo que Iivonen (1987: 242) conoce como pregunta-eco.

Por lo demás, uno de los rasgos que parecen caracterizar a los enunciados interrogativos es la marcada altura tonal con que se inicia la frase, que comenzando en la primera sílaba continúa hasta la palabra más relevante del enunciado, porque, según Hirvonen (1970: 31), «By the location of this fall the speaker probably indicates to his listener the matter that he particulary wants to inquire about». Después (o durante) la primera sílaba de esa palabra importante semánticamente - «propos»- el tono desciende rápidamente, siendo el resto exactamente igual que en el enunciado declarativo. En palabras de Hirvonen,

General questions have one characteristic feature: a high initial pitch. That high pitch begins on the head and continues until the most important word of the sentence. After (or during) the first syllable of the propos the pitch falls rapidly, and the rest of the pattern is quite like the normal breath-group (1970: 31).

Ese perfil melódico que la frase interrogativa presenta en finés con los tonos altos concentrados al comienzo parecen reclamar en opinión de Hirvonen (1970: 49) una puntuación distinta, más acorde con la realidad acústica, en la 
que el signo de interrogación apareciese —como se hace en español— también al principio de la frase:

[...] it would really be more sensible in Finnish, too, to use punctuation at the beginning of the sentence as well, as an analogous attention-getting device, like «Tuleks sä töihin huomenna?" [¿Vienes mañana a trabajar?] (1970: 49).

Hirvonen concluye que, en todo caso, las interrogativas se distinguen claramente de los enunciados declarativos completos. A conclusiones similares llegan otros estudiosos como livonen (1978), o Wiik (1981: 118-119), quien afirma: «es evidente que en finés la frase interrogativa y la afirmativa tienen diferente entonación» porque el nivel tonal medio es más alto en las interrogativas?

También las preguntas pronominales parecen estar caracterizadas por un tono inicial alto (Hirvonen 1970: 33). El tono cae después de la primera o segunda sílaba porque, según Karlsson (1983: 172), el interés de la pregunta está concentrado en la partícula interrogativa inicial. El resto de la curva sigue la del enunciado declarativo. En palabras de Hirvonen,

particular questions are also characterized by a high initial pitch. Since the initial interrogative word alone normally forms the propos, the pitch falls after the first or second syllable. The rest of the pattern is like the normal breath-group (1970:33).

En la entonación de los enunciados imperativos el tono de la primera sílaba tónica es un poco más bajo que en los enunciados interrogativos, pero mantiene por lo demás un nivel tonal más alto que en las aseverativas (Wiik 1981: 119), y presenta una gran similaridad con el esquema melodico de aquéllos. Así, Hirvonen dice:

It seems fairly certain that imperatives don't constitute a separate intonational category [...] it can be said that imperatives differ from the basic breath-group but not from questions (1970:37).

Por lo demás, los perfiles descritos por la curva melódica para expresar otros valores están tan próximos que es difícil sistematizar. Así, Karlsson afirma:

7 WuK (1981: 118) pone como ejemplo la frase del finés «Olit să eilen ulkona» (tú saliste ayer), que con un nivel tonal alto se entiende como pregunta, mientras que si se enuncia con un tono más bajo será interpretada como aseveración. 
[...] en la práctica, los esquemas melódicos están tan próximos que no pueden diferenciarse. Esto es lo que ocurre con matices tan sutiles como invitación, petición, recomendación, mandato cortés, susceptibles todos ellos de expresión prosódica. Su interpretación debe hacerse en última instancia de acuerdo a la situación concreta en que se enuncia. No pueden formularse normas generales sobre ellas (1983: 174).

\section{SimilltudeS Y DIFERENCIAS ENTRE LAS DOS LENGUAS}

La descripción de los sistemas entonativos del español y el finés que acabamos de realizar nos permite establecer los principales puntos de contacto, así como las divergencias que existen entre ambos; en síntesis:

1. Los especialistas que analizan la importancia de los rasgos físicos de la entonación de cada una de las lenguas coinciden en conceder la mayor importancia al tono, sin negar la importancia de intensidad y cantidad.

2. La amplitud tonal del intervalo en que se desarrollan los movimientos de la voz en el español estándar parece ser mayor que la correspondiente en finés.

3. La entonación del español presenta mayor variedad de inflexiones que la del finés

4. Los enunciados interrogativos - tanto absolutos como pronominales-, así como los imperativos, están caracterizados en finés por el empleo de tonos altos -en su tramo inicial, especialmente- que podrían constituir índices para el reconocimiento de la melodía.

5. El finés dispone de una serie de partículas — «ko», «pa», «kin», «hän»- con valores tan diversos como pregunta, insistencia, confirmación, etc., que cumplen el valor que en español es desempeñado normalmente por la entonación.

6. Los tres tonemas fonologicamente pertinentes en el español -cadencia, suspensión y anticadencia-- poseen valor distintivo en finés sólo en los dos primeros casos: puede actuar como única marca de que el hablante ya ha concluido su intervención en frases con cadencia, o de que tiene intención de continuar hablando cuando concluye en suspensión. El tonema de anticadencia, sin embargo, carece de ese valor porque la pregunta en finés ya viene marcada obligatoriamente por medios morfologicos - los sufijos -«ko» y -«kö», la conjunción disyuntiva «vai», 
etc. - que hace redundante el uso de la inflexión final ascendente de la línea melodica.

7. La inflexión ascendente en finés puede cumplir valores expresivos - -sorpresa, apelación cortés, etc.- pero no lingüísticos. Su escaso uso para expresar pregunta, limitado a algunas variantes dialectales o a la lengua corriente de algunas grandes ciudades por influencia foránea, hace que sea considerada extraña al finés.

8. La entonación cumple en finés, al igual que en español, las funciones lingüísticas integradora y delimitadora. El importante valor distintivo que la entonación desempeña en español se cumple sólo parcialmente en finés, al oponer en esta última lengua enunciados acabados frente a los que no lo están, pero no afirmativos e interrogativos en los que las diferencias ya vienen expresadas morfologicamente.

9. En otros niveles, la entonación cumple en ambas lenguas las funciones identificadora, expresiva y sociolingüística.

10. En suma, la entonación en finés parece desempeñar un papel mucho menos importante que en español.

3. TEST DE DISCRIMINACIÓN DE LOS TONEMAS DEL ESPAÑOL

\subsection{Criterios de Selección}

A la hora de confeccionar el material objeto de análisis se han tenido en cuenta las siguientes premisas:

1. Para el estudio de la discriminación de los distintos patrones entonativos se han seleccionado frases que representen los cinco tonemas que Navarro Tomás (1971: 6-7) distingue para el español: cadencia, anticadencia, suspension, semicadencia y semianticadencia. Los tonemas menores de semicadencia y semianticadencia son sometidos también a prueba para saber si nuestros alumnos asocian esas variantes con los tonemas extremos de anticadencia y cadencia o, por el contrario, con el de suspensión. Resulta obvio que lo importante es que el estudiante perciba aquellas diferencias que tienen relevancia desde el punto de vista fonológico - debe ser capaz de distinguir una inflexion ascendente de una cadencia, o el tonema que caracteriza a un enunciado inacabado del que acompaña a uno completo-, pues una interpretación equivocada significaría un mensaje erróneo. No podemos negar, sin embargo, el interés que representa para nosotros conocer cuál es el 
grado de comprensión y la interpretación de los tonemas menores. Seguimos, en definitiva, las directrices marcadas por Navarro Tomás cuando dice:

Ha sido corriente en los estudios de entonacion [...] agrupar simplemente las unidades melódicas [...] bajo los dos tipos de inflexión descendente $o$ ascendente. Se han reducido a estos dos únicos tipos no sólo las unidades de la elocución enunciativa, sino también las de la entonación interrogativa y emocional. Un análisis más sistemático de la materia llevará dentro de cada uno de esos campos a diferenciar las unidades secundarias de las principales, y a determinar la serie de sintonemas de cada lengua y forma y función de cada tipo, base indispensable para poder comparar desde este punto de vista unas lenguas con otras (1974: 53-54).

2. La frase cuya entonación debe ser identificada por los estudiantes deberá contener sólo un grupo fónico. Aquéllas que contienen los tonemas de semicadencia y semianticadencia, por tanto, han sido separadas, desechando el resto del enunciado grabado.

3. De cara a facilitar el tratamiento y la exposición de los datos, así como la corrección y el análisis de los resultados, cada uno de los cinco tonemas ha sido dotado de un código (una o dos letras) que responden al esquema siguiente:

Cadencia (C)

Anticadencia (A)

Suspensión (SU)

Semicadencia (SC)

Semianticadencia (SA)

4. Cada uno de los tonemas aparece en tres frases diferentes, por lo que contamos con un corpus de quince frases.

5. Los tonemas y las frases que los representen serán los siguientes:

CADENCIA (C)

Tres enunciados afirmativos simples:

a) Quieren aprender español (C).

b) La vi por la tarde (C).

c) Estoy acostumbrado (C). 
ANTICADENCIA (A)

Tres enunciados interrogativos absolutos:

a) ¿Estuvieron conversando? (A).

b) ¿Ya ha acabado? (A).

c) ¿Me han llamado? (A).

\section{SUSPENSIÓN (SU)}

Para el tonema de suspensión se han elegido oraciones de tres grupos en las que la rama tensiva o prótasis consta de un primer elemento acabado en suspensión y un segundo en anticadencia, seguido de la apódosis que acabará en cadencia.

a) El sobrino de Manolo ( $\mathrm{SU}$ ), con enorme valentía, lo defendió ante los otros.

b) La clase de civilización ( $\mathrm{SU}$ ), como de costumbre, será los martes.

c) El sábado próximo (SU), si no ocurre nada, iremos a veros.

\section{SEMICADENCIA (SC)}

Para la semicadencia hemos elegido:

a) Una enumeración simple enunciativa, independiente y cerrada, sin ningún carácter expresivo especial, en la que los miembros de la enumeración van seguidos de semicadencia, a excepción de los dos últimos:

El sueco, el noruego (SC), el danés, el islandés y el finlandés forman los llamados pueblos nórdicos.

b) Una frase que sirve de antecedente a una oración de relativo que, intercalada en la prótasis, interrumpe el sentido de la principal:

Los compañeros de mi hermano (SC), que son de Zaragoza, vendrán a visitarnos.

c) El primer complemento circunstancial de una serie complementaria al principio de la frase en la que es frecuente el descenso en semicadencia:

Estando ya jubilado (SC), sin ninguna afición y con poca salud, le faltaban las ganas de vivir.

\section{SEMIANTICADENCIA (SA)}

Para la selección de los ejemplos de oraciones con semianticadencia nos hemos servido de:

a) El segundo grupo - primero de la apódosis- de una oración de tres, cuyo primer elemento - prótasis- acaba en anticadencia y el último en cadencia: 
En el piso de arriba, se hace los fines de semana (SA) un ruido insoportable.

b) Un grupo complementario, intercalado en la apódosis:

Por esa ventana me escapaba yo, cuando era pequeño (SA), a jugar con mis amigos.

c) Una enumeración distributiva: serie de dobles núcleos en las que el primer grupo acaba en semianticadencia; el segundo, en cadencia:

El pasillo, oscuro; la habitación del niño (SA), demasiado pequeña; la cocina, estrecha...

\subsection{Grabación del material lingüístico}

Seleccionadas las frases que serían objeto de análisis, y dotadas del código correspondiente, procedimos a distribuirlas en orden aleatorio. Con el fin de que la melodía del grupo fónico elegido respondiese lo más fielmente posible al patrón entonativo apropiado, para la lectura se tomaron las frases en su totalidad. Con posterioridad fue eliminada la parte de la frase que no interesaba, conservando s6́lo el grupo fónico elegido. El registro de las frases se llevó a cabo en un laboratorio de grabación, sobre banda magnética, con un aparato $R e$ vox $B 77 M k I I$ y un microfono Audio-Technica AT813, en condiciones técnicas y acústicas 6ptimas. Las frases fueron leídas por un español, estudiante universitario natural de Salamanca, y con residencia habitualmente en esa misma ciudad, que en el momento de la prueba se encontraba en Finlandia realizando estudios del último curso de carrera en la Escuela Superior de Comercio de Turku, en el marco del Programa Erasmus. Teniendo en cuenta, sin embargo, que los informantes en la prueba serían alumnos que ya poseían algunos conocimientos de español, con lo que fácilmente podrían comprender el significado de las frases, y con ello deducir su valor entonativo, decidimos someter las frases registradas a un proceso de manipulación que enmascararía su significado. Para ello recurrimos, primeramente, al DSP Sona-Graph, modelo 5500 de la casa Kay Elemetrics de New Jersey. Cada una de las frases registrada fue introducida en el sonógrafo. Seguidamente procedimos a trasladarla a un ordenador. El programa de análisis y síntesis utilizado fue el LPC Parameter Manipulation / Synthesis, modelo 5635, versión $1 X$. Una vez introducida en el ordenador fueron alterados el primer y segundo formante de cada frase, sin realizar modificaciones en la línea melódica ni en los otros rasgos suprasegmentales. Se trataba de distorsionar la información segmental sin alterar la suprasegmental. A continuación, fueron devueltos al Sono-Graph 5500, analiza- 
dos en pantalla, y escuchadas y comparadas las versiones original y alterada. Cuando la versión manipulada enmascaraba suficientemente el significado de la frase, se procedía a su grabación en banda magnética y se imprimía el sonograma correspondiente.

\subsection{La prueba}

Antes de efectuar la prueba definitiva, por ser varias las dudas que se nos planteaban respecto a la forma más adecuada de realizar el test, y convencidos de los buenos resultados que nos podría reportar, decidimos realizar un experimento piloto con el que pretendíamos, entre otras cosas, elegir la forma más adecuada de presentar gráficamente los diferentes tonemas con los que los alumnos deberían identificar los grupos fónicos elegidos. De esta forma, siguiendo la opinión de los propios estudiantes, se optó por tres flechas (ascendente, descendente y horizontal) en lugar de los signos de puntuación (signo de interrogación, punto y coma) que en principio se había pensado. En segundo lugar, queríamos comprobar si efectivamente la presentación de las frases bajo una u otra forma - versión original o alterada - tenía incidencia en los resultados. Tras la realización de la prueba pudimos comprobar que el número de errores disminuía cuando se les hacía escuchar las frases en su version original: comprender el significado de la frase ayudaba a una mejor identificación de su patrón melódico. Vimos, por tanto, la conveniencia de manipular las frases cuya entonación debía ser discriminada. No desechamos, sin embargo, las frases en su grabación original; decidimos someterlas también a prueba, pues ello nos permitiría comparar los resultados y ver similitudes y diferencias entre ambas. Finalmente, reducir la representación de los cinco tonemas a sólo tres símbolos demostró ser suficiente. Ello, además, presentaba la ventaja de permitimos saber si los tonemas menores eran identificados con el de suspensión, o bien con los tonemas extremos.

\subsubsection{Los informantes}

Para la prueba nos servimos de estudiantes que seguían el nivel umbral de español (curso de sesenta horas; cuatro semanales), durante una de las últimas clases del curso. El grupo estaba formado por catorce chicas y seis chicos. Todos ellos eran estudiantes universitarios y poseían - a juzgar por las respuestas que figuraban al principio de la hoja de corrección- muy buenos conocimientos de lenguas extranjeras: la mayoría tenía un excelente conocimiento del 
inglés, y muy bueno o bueno del sueco; tres de ellos conocían muy bien -otros diez, en alguna medida- el alemán. Once habían seguido cursos de francés: ocho de ellos con conocimientos regulares; tres, muy buenos. Las otras lenguas sólo se conocían en muy escasa medida: dos, italiano; uno ruso; y uno, portugués. Por su lugar de procedencia, once eran del suroeste de Finlandia - Turku o proximidades - y el resto de otras zonas del país; mayoritariamente, del sur.

\subsubsection{Corrección de los Tests}

Para la correción de los tests debían establecerse previamente unos criterios de valoración que determinasen con precisión qué identificaciones deberían ser consideradas correctas y cuáles no. Por ello, teniendo en cuenta las observaciones anteriormente hechas sobre la entonación del español en lo que a las tres inflexiones tonales pertinentes se refiere, acordamos considerar errores las confusiones producidas entre los tres tonemas mayores - ascendente, descendente y horizontal-; es decir: una anticadencia percibida como cadencia $o$ suspensión; una cadencia, como suspensión o anticadencia, etc. En lo relativo a los tonemas menores, teniendo en cuenta la falta de unanimidad que impera entre los estudiosos a la hora de atribuir su filiación como variantes - unos los agrupan con los tonemas extremos de anticadencia o cadencia; otros, con el de suspension - decidimos dar por válidas ambas respuestas. Se considerarían errores también las identificaciones que confundiesen los tonemas extremos entre sí; es decir: la semianticadencia percibida como cadencia, o la semicadencia como anticadencia.

\subsection{Resultados}

\subsubsection{Valoración global}

En el cuadro 1 puede observarse el número y porcentaje de identificaciones erróneas producidas en el test. Además de las correspondientes a los tonemas de cadencia, anticadencia y suspensión, en él aparecen representadas las confusiones producidas en los tonemas menores de semicadencia y semianticadencia.

Comencemos por la versión cuya información segmental ha sido alterada. Puede apreciarse que el mayor número de errores se registra en los tonemas de cadencia y suspension. La inflexión final de cadencia se identifica errónea- 
mente en 39 ocasiones, lo que se significa el $65 \%$ del total de los 60 casos analizados; el tonema de suspensión, a su vez, es interpretado como cadencia o anticadencia en 35 casos (58,3\% del total de su serie). Los tonemas menores son los que parecen representar las menores dificultades, al ser identificada la semicadencia como anticadencia en sólo 4 casos $(6,7 \%)$, mientras la semianticadencia se percibe como cadencia en tan sólo 1 de los 60 casos analizados.

Por lo demás, existe una gran uniformidad entre los resultados de nuestros informantes. Los errores, en efecto, se concentran en los tonemas de cadencia y suspensión. Aquellos alumnos con un alto nivel de aciertos en sus respuestas cometen sus únicos errores en aquellas series.

Se observa, además, que las confusiones se producen mayoritariamente entre tonemas proximos: la cadencia registra el mayor tipo de desvíos hacia la suspensión, siendo aislados los casos en que se identifica erróneamente como tonema ascendente. El tonema horizontal, por su parte, es confundido principalmente con el de cadencia.

En lo que a la anticadencia se refiere, los pocos casos de identificaciones erróneas producidas se realizan indistintamente por confusión con el tonema de suspensión o cadencia.

Los tonemas menores, por último, acusan una dificultad muy pequeña en el caso de la semicadencia y ninguna en el de la semianticadencia, al producirse sólo un error en cada una de las dos versiones, de nulo valor estadístico.

\subsubsection{Versión original y Versión manipulada}

El número de identificaciones erróneas que nuestros informantes han cometido en el test de discriminación de la entonación acusa visibles diferencias entre la versión manipulada y la original. Si observamos el gráfico 1, donde se comparan los errores producidos en los dos tests, podemos hacernos una idea general de los tonemas más problemáticos y las diferencias más relevantes entre los resultados de ambas versiones.

Frente a las 86 identificaciones erróneas, con una media del $28,6 \%$, registradas en el test en el que los informantes han escuchado la versión manipulada, aparecen 54 ( $18 \%$ de media) en la versión original. ¿Cuál es la razón de estas diferencias? Si tenemos en cuenta que la manipulación de las frases registradas sólo ha afectado a los segmentos — han sido alterados sólo los dos primeros formantes - pero los medios suprasegmentales han permanecido intactos, así como el resto de las circunstancias en las que los tests han sido llevados a cabo - las dos pruebas han sido realizadas, consecutivamente, en la misma sesion, con iguales informantes, partiendo de las mismas grabaciones, 
idéntico material de reproducción y circunstancias de audición, igual procedimiento seguido, así como el mismo formulario de respuesta- sólo la comprensión del mensaje de las frases inalteradas puede dar explicación a estas divergencias. Los sujetos de la prueba, alumnos del primer año de español al final del curso en el momento de realizar el test ya poseían unos conocimientos mínimos de español que les capacitaba para entender los enunciados sencillos presentados en la prueba. Bien es verdad que cada una de las frases sometidas a audición habría podido estar dotada de cualquiera de los otros tonemas analizados, pero también lo es que las posibilidades de interpretar correctamente un tonema serán mayores cuando se comprende el valor aislado de todas o la mayoría de las palabras presentes en la frase y ésta posee una estructura fácilmente interpretable como completa -es el caso de la vi por la tarde - u otra mucho más susceptible de ser comprendida como inacabada: estando ya jubilado...

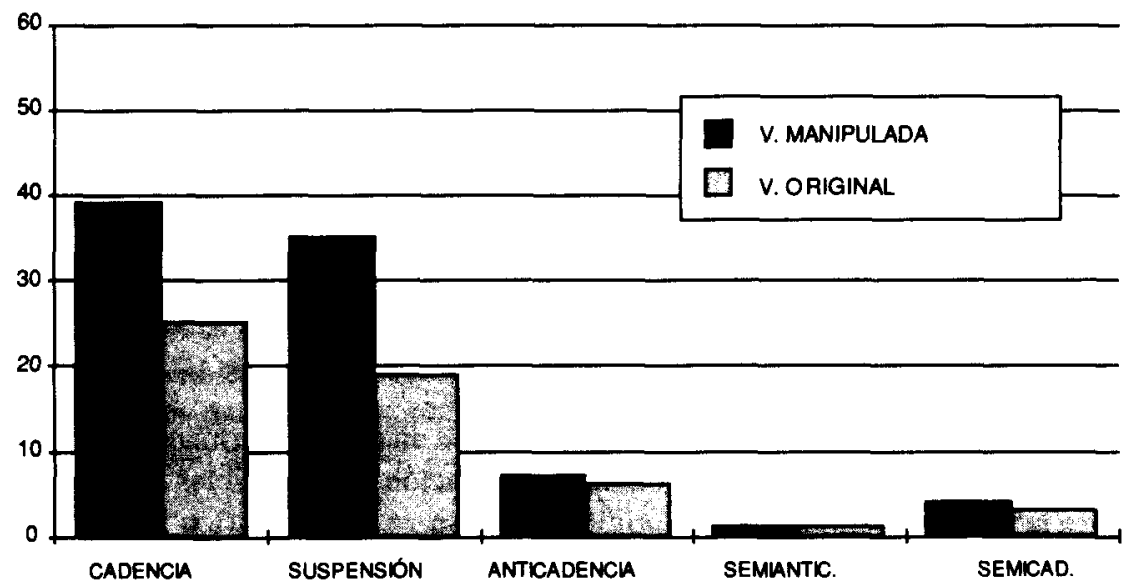

Gráfico 1. Comparación de los errores de discriminación de la entonación en las versiones original y manipulada.

Por lo demás, parece existir un gran paralelismo entre los resultados de ambos tests. Observamos, en efecto, que el mayor número de errores se produce en ambos tests en el tonema de cadencia - 39 y 25 errores-, seguido de la suspensión - 35 y 19-, la anticadencia -7 y 6 -, la semicadencia 4 y 3 - y, finalmente, la semianticadencia, donde en ambos casos se produce un solo error. El tipo de errores producido, así como la magnitud de aqué- 
llos y los tonemas motivo de confusión son objeto de atención en el apartado siguiente.

3.5.3. Errores en la identificación de los tonemas: tipo y proporción de las desviaciones

A las similitudes ya apuntadas en apartados anteriores debe añadirse la que se produce en el tipo y proporción de las desviaciones que los diferentes tonemas analizados sufren en las identificaciones incorrectas de ambos tests. Las confusiones con otro tonema son en la mayor parte de los casos parecidas: la cadencia se interpreta como suspensión en 33 de los 60 casos en la versión manipulada, mientras que en la original la identificación incorrecta con ese tonema se produce en 22 ocasiones; las identificaciones erróneas del tonema descendente con el de anticadencia se produce en 6 y 3 casos, respectivamente. El tonema horizontal se desvía en 23 ocasiones hacia el tonema de cadencia y en 12 hacia el ascendente, mientras que en la versión alterada estas proporciones son de 18 y 1 , respectivamente. La anticadencia, que registra muchos menos errores que los tonemas extremos, no parece, sin embargo, arrojar grandes diferencias entre una y otra versión, pues se identifica erróneamente en 7 casos en la versión manipulada -4 desvíos hacia la suspensión y 3 hacia la cadencia- y en 6 en la original: 2 confusiones con el tonema horizontal y 4 con el de cadencia.

¿Cómo dar explicación a estos resultados? ¿Por qué se produce el mayor tipo de errores en la identificación de los tonemas de cadencia y suspension? ¿Cuál es la causa de un tan alto porcentaje $-65 \%$ y $58,3 \%$, respectivamentede confusiones? ¿Cómo explicar que estos tonemas se confundan entre sí -33 de las 39 interpretaciones erróneas tienen como destino la suspensión, y 23 de las 35 supensiones son interpretadas como cadencias- en la mayor parte de los casos?

El finlandés no está acostumbrado, evidentemente, a prestar mucha atención a las inflexiones melódicas. Su lengua dispone de elementos morfológicos que hacen prácticamente innecesaria la presencia de la melodía, que más bien parece desempeñar una función expresiva que linguística. La mayor parte de las confusiones de identificación se realiza con tonemas próximos - suspensión con cadencia y cadencia con suspensión, principalmente-, lo que indica una cierta aproximación, pero sin llegar a percibir con exactitud la dirección del tonema. La diferencia de tono en esos casos resulta excesivamente sutil para el oído medio finlandés. Sólo en 6 ocasiones en la versión manipulada -3 en la original- de los 60 casos de cadencia, el tonema es interpretado como ascen- 
dente. Las proporciones de confusión del tonema ascendente con el de cadencia son aún menores, al producirse sólo en tres y cuatro ocasiones, respectivamente. Por último, los tonemas menores confirman la tendencia ya apuntada para los otros, al ser interpretada la semicadencia como ascendente en cuatro casos, y el tonema de semiantianticadencia como descenso del tono en sólo un caso.

No dejan de llamar la atención, sin embargo, los resultados —en este caso muy diferentes a lo que habíamos previsto- producidos para el tonema de anticadencia. Las identificaciones incorrectas en este caso son mucho menores que las producidas en los de cadencia y suspensión. Aunque el tonema ascendente que caracteriza a las interrogativas absolutas del español no puede considerarse, como ya sabemos, un tonema propio del finés, y el finlandés medio no está, por tanto, familiarizado con ese tipo de inflexión, la diferencia de tono característica del final de la frase es suficientemente grande para ser percibida con un elevado grado de aciertos: $\mathbf{8 8 , 3 \%}$ en la versión manipulada y $90 \%$ en la original.

El cuadro 1 con las identificaciones erróneas totales y el tipo de desviaciones proporcionará una idea más clara del tipo y magnitud de los errores producidos y posibilitará una más rápida comparación de los resultados de ambos tests.

\subsubsection{Identificación de los tonemas menores}

Los tonemas menores, como puede apreciarse en el cuadro 2, son identificados sin problemas. Debe destacarse la gran uniformidad que arrojan los dos tests: tanto las frases manipuladas como las que fueron presentadas en su versión original registraron el mismo número de errores: sólo uno en el caso de la semianticadencia y cuatro en el del tonema de semicadencia.

A la hora de adscribir semicadencia y semianticadencia a un tonema determinado, los especialistas presentan divergencia de opiniones, pues mientras unos prefieren verlos como variantes de cadencia y anticadencia, respectivamente, otros prefieren considerarlos realizaciones particulares del tonema horizontal. Entre los primeros se encuentran Quilis y Fernández:

Al tonema fonologicamente ascendente, corresponderán, en el plano fonético, la anticadencia y la semianticadencia [...] al tonema fonológicamente descendente, corresponderán, en su realización, dos variantes: la cadencia y la semicadencia $(1979: 165)$.

También Alarcos Llorach (1994: 52) es de la misma opinion: «la semicadencia y la semianticadencia [...] son más bien variantes de la cadencia y la an- 


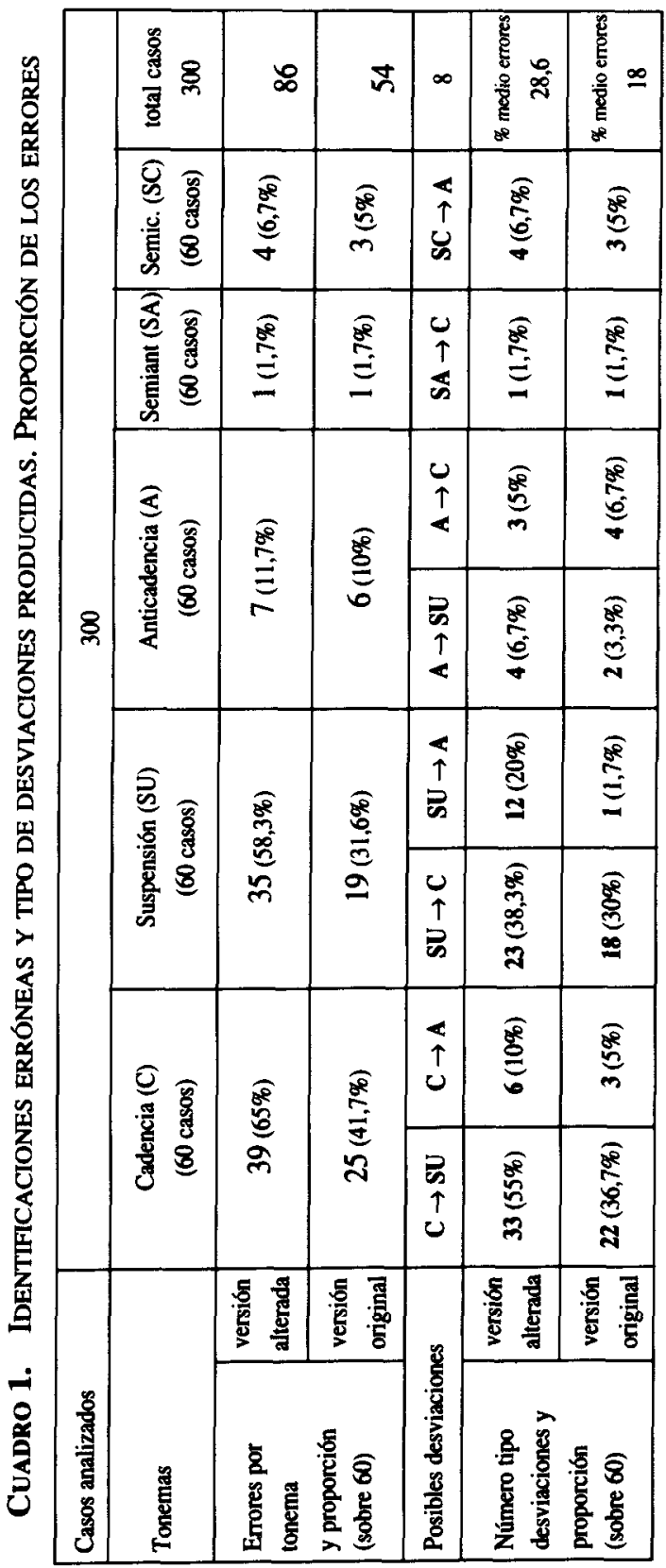


ticadencia». Alcina y Blecua (1975: 471), sin embargo, afirman: «los contrastes menores aparecen como variantes de $/ \rightarrow / »$.

Los resultados de nuestro test parecen confirmar, sin embargo, la opinión de los primeros, pues en el test de identificación —versión manipulada - en 47 casos $(78,3 \%)$, de un total de 59 respuestas correctas, se identifica la semianticadencia como anticadencia, mientras que sólo en 12 casos aquélla es adscrita a la suspension. Los porcentajes son aún más elocuentes en el test con la versión original, pues el $88,3 \%$ de los casos (53 identificaciones) asocia semianticadencia con el tonema ascendente.

Los resultados relativos a la semicadencia, aunque menos contundentes, parecen también confirmarnos la conveniencia de asociar los tonemas menores con los extremos, pues en 32 ocasiones - tanto en la manipulada como en la original- de un total de 56 respuestas correctas la semicadencia es identificada con el tonema descendente.

\section{Cuadro 2.}

\begin{tabular}{|c|c|c|c|c|c|c|c|c|}
\hline & \multicolumn{8}{|c|}{ IDENTIFICACIONES DE LOS TONEMAS MENORES } \\
\hline & \multicolumn{8}{|c|}{ VERSIÓN ALTERADA (120 casos) } \\
\hline & \multicolumn{6}{|c|}{ Aciertos: $115(95,8 \%)$} & \multicolumn{2}{|c|}{ Enores: $5(4,2 \%)$} \\
\hline & \multicolumn{3}{|c|}{ Semianticadencia (SA/ 60 casos) } & \multicolumn{3}{|c|}{ Semicadencia ( $\mathrm{SC} / 60$ casos) } & Semiant. & Semicad. \\
\hline & $\mathrm{SA} \rightarrow \mathrm{SU}$ & $S A \rightarrow A$ & total & $\mathrm{SC} \rightarrow \mathrm{SU}$ & $\mathrm{SC} \rightarrow \mathrm{C}$ & total & $\mathrm{SA} \rightarrow \mathrm{C}$ & $\mathrm{SC} \rightarrow \mathrm{A}$ \\
\hline no identif. & 12 & 47 & 59 & 24 & 32 & 56 & 1 & 4 \\
\hline \multirow[t]{5}{*}{$\%$ sobre 60} & $20 \%$ & $78,3 \%$ & $98,3 \%$ & $40 \%$ & $53,3 \%$ & $93,3 \%$ & $1,7 \%$ & $6,7 \%$ \\
\hline & \multicolumn{8}{|c|}{ VERSIÓN ORIGINAL (120 casos) } \\
\hline & \multicolumn{6}{|c|}{ Aciertos: $116(96,7 \%)$} & \multicolumn{2}{|c|}{ Enores: $4(3,3 \%)$} \\
\hline & \multicolumn{3}{|c|}{ Semianticadencia ( $\mathrm{SA} / 60$ casos) } & \multicolumn{3}{|c|}{ Semicadencia ( $\mathrm{SC} / 60$ casos) } & Semiant. & Semicad. \\
\hline & $\mathrm{SA} \rightarrow \mathrm{SU}$ & $S A \rightarrow A$ & total & $\mathrm{SC} \rightarrow \mathrm{SU}$ & $\mathrm{SC} \rightarrow \mathrm{C}$ & total & $\mathrm{SA} \rightarrow \mathrm{C}$ & $\mathrm{SC} \rightarrow \mathrm{A}$ \\
\hline no identif. & 6 & 53 & 59 & 17 & 40 & 57 & 1 & 3 \\
\hline$\%$ sobre 60 & $10 \%$ & $88,3 \%$ & $98,3 \%$ & $28,3 \%$ & $66,7 \%$ & $95 \%$ & $1,7 \%$ & $5 \%$ \\
\hline
\end{tabular}




\section{Conclusión}

El elevado porcentaje de errores que en ambas pruebas se registran, junto a la coincidencia en el tipo de tonemas, proporciones en las confusiones para cada uno de aquéllos e inflexiones melódicas destino de las identificaciones erróneas, constituye el reflejo de una habilidad sólo desarrollada parcialmente por nuestros alumnos, consecuencia sin duda de la presencia en su lengua de elementos morfológícos que restan importancia a ese rasgo suprasegmental. El menor número de confusiones producido en la identificación de los tonemas de la versión original, por lo demás, hace patente la ayuda que la información segmental proporciona a la hora de interpretar correctamente la melodía.

\section{BIBLIOGRAFIA}

AALtonen, O., y WIIK, K. (1979): «Suomen jatkuvuuden intonaatiosta», en P. Hurme (ed.), Puheentutkimuksen alalta 1. Fonetiikan päivät, Jyväskylän yliopiston suomen kielen ja viestinnän laitoksen julkaisuja, 18, 23-33.

AHONEN-MÄKelÄ, R. (1975): Ilo puhua. Ajatuksia puheilmaisusta, Helsinki, SKS.

Alarcos, LloRACH, E. (1994): Gramática de la lengua española, Madrid, Espasa Calpe.

Alcina Franch, J., y BleCUA, J.M. (1975): Gramática española, Barcelona, Ariel.

GILI GAYA, S. (1975): Elementos de fonética general, Madrid, Gredos.

HAKULINEN, L. (1979): Suomen kielen rakenne ja kehitys, Helsinki, Otava.

HeLIN, J. y otros (1985): «Haloo-puhelinpuheen prosodia», en Olli Aaltonen-Timo

Hulko (eds.), XIII Fonetiikan päivät, Turku, Turun yliopiston suomalaisen ja yleisen kielitietee laitoksen julkaisuja, 26: 55-64.

HIRVONEN, P. (1970): Finnish and English Communicative Intonation, Turku, Turun yliopiston fonetiikan laitoksen julkaisuja.

IIVONEN, A. (1978): «Is There Interrogative Intonation in Finnish? en Gårding (ed.), 43-53.

- (1980): «Suomen kysymyslauseiden prosodiikan määräytyvyydestä», en O. Iko-

la (edit.), Congressus Quintus Internationalis Fenno-Ugristarum, 6, Turku, Suomen Kielen Seura, 101-107.

Irvonen, A. y otros (1987): Puheen intonaatio, Helsinki, Gaudeamus.

KALLIOINEN, V. (1968): «Suomen kysymyslauseen intonaatiosta», Virittäjä 72, 35-54. KARLSSON, F. (1981): Suomi vieraana kielenä, Helsinki, WSOY.

-. (1983): Suomen kielen äänne- ja muotorakenne, Helsinki, WSOY.

Lehtonen, J. (1971) Puheen sävelkulun merkitys ja muoto. Alustavia kokeita ekspresiivisen intonaation analysoimiseksi, Jyväskylä, Jyväskylän yliopiston suomen kielen laitoksen julkaisuja. 
LehtovaARA, J. (1978): Peruskoulun kolmasluokkalaisten englannin kielen ääntämistaidosta Tamperen yliopiston kasvatustieteen laitos, Tampere.

Lieberman, P. (1967): Intonation, Perception, and Language, Cambridge, Mass.

LIEKO, A. (1992): Suomen kielen fonetiikkaa ja fonologiaa ulkomaalaisille, Loimaa, Finn Lectura.

LORENZO, E. (1994): «Sobre la enseñanza de español a extranjeros», en Jesús Sánchez Lobato - Isabel Santos Gargallo (eds.), Problemas y métododos en la enseñanza del español como lengua extranjera, Madrid, SGEL, 9-19.

MONOLA, T. (1976): Aino Kallaksen ja Marja-Liisa Vartion proosarytmin vertailua. Kolmen proosakatkelman rytmianalyysi, Helsinki, Helsingin yliopiston fonetiikan laitoksen julkaisuja, 28A y 28B.

NavarRo TOMÁs, T. (1971): «Diptongos y tonemas», Thesaurus. Bol. Inst. Caro y Cuervo XXVI: $1-10$.

NAVARRo TOMÁs, T. (1974): Manual de entonación española, Madrid, Guadarrama.

PENTTLLA, A. (1958): «Intonaatiotutkimuksia», Virittäjä, 61: 1-19.

Quils, A. (1981): Fonética acústica de la lengua española, Madrid, Gredos.

QULIS, A., y FERNÁNDEZ, J.A. (1979): Curso de fonética y fonología españolas, Madrid, CSIC.

SuOMI, K. (1975): «Puhesynteesi ja intonaatio» Fonetiikan paperit, 1/75: 43-44.

WIIK, K. (1981): Fonetiikan perusteet, Porvoo-Helsinki-Juva, WSOY. 